Esta revista forma parte del acervo de la Biblioteca Jurídica Virtual del Instituto de Investigaciones Jurídicas de la UNAM

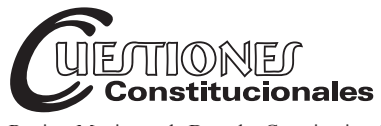

Revista Mexicana de Derecho Constitucional

Núm. 44, Enero-Junio 2021

ISSN (versión electrónica): 2448-4881

\title{
Igualdad y acciones afirmativas: el caso del acceso prioritario a los Centros de Atención y Cuidado Infantil para la Ciudad de México (CACI)
}

\author{
Equality and affirmative actions: The case \\ of priority access to the Child Care and Attention \\ Centers of Mexico City (CACI)
}

\author{
Francisco Alejandro \\ OLMOS DE LA TORRE*
}

RESUMEN: Este comentario ofrece una crítica a la decisión de la Suprema Corte de Justicia de la Nación, tomada en la acción de inconstitucionalidad 215/2020, de desestimar un argumento formal de igualdad, por el cual se buscaba la inclusión de los hombres en una acción afirmativa dirigida exclusivamente a las mujeres. Salvo el ponente del asunto, los ministros y ministras consideraron que este planteamiento incluso invisibilizaba el problema de las mujeres y fortalecía la cultura machista. Parecieran ponerse en conflicto dos visiones de la igualdad: una formal y otra estructural. Desde una óptica conciliadora, el autor pretende mostrar la razonabilidad del planteamiento formal de igualdad, dado que no compromete en este
ABSTRACT: This comment criticizes the Supreme Court of Justice of the Nation decision, taken in the "acción de inconstitucionalidad" 215/2020, of rejecting a formal argument of equality, which implies the inclusion of men, in an affirmative action in favor only of women. Except for the ponent, the justices considered that this approach even made the problem of women invisible and strengthened the "macho" culture. Two visions of equality seem to conflict: one formal and the other structural. From a conciliatory point of view, the author tries to expose the reasonableness of the formal equality approach, arguing why it does not compromise women, as a socially excluded group, in this case.

\footnotetext{
Abogado por la Universidad de Guadalajara y Maestro en Derecho y Derechos Humanos y Democracia por la Universidad Nacional Autónoma de México y la Facultad Latinoamericana de Ciencias Sociales. Secretario de Estudio y Cuenta de la Suprema Corte de Justicia de la Nación. México. Correo electrónico:folmost@mail.scjn.gob.mx ORCID: 0000-0003-2486-1625.
} 
Esta revista forma parte del acervo de la Biblioteca Jurídica Virtual del Instituto de Investigaciones Jurídicas de la UNAM

caso a las mujeres, como grupo socialmente excluido.

Palabras clave: acciones afirmativas, mujeres, constitucionalidad, igualdad formal, igualdad estructural, infrainclusividad, suprainclusividad, test de igualdad.
Keywords: affirmative actions, women, constitutionality, formal equality, structural equality, infra-inclusiveness, over-inclusiveness, equality test.

SUMARIO: I. El caso. II. La crítica. III. Bibliografía.

\section{EL CASO}

En sesión de 8 de marzo de 2021, Día Internacional de la Mujer, el Pleno de la Suprema Corte analizó la acción de inconstitucionalidad 215/2020, en la que la Comisión Nacional de los Derechos Humanos (CNDH) controvirtió la validez de una acción afirmativa que beneficia exclusivamente a las mujeres. Se trata de la medida prevista en el artículo 27, párrafo segundo, fracciones I, II y III, de la Ley que regula el funcionamiento de los CACI, ${ }^{1}$ y que establece la prioridad que tienen exclusivamente las "madres" para la admisión de sus hijas e hijos en estos centros, bajo ciertos supuestos, que son: a) tener entre 12 y 22 años 11 meses de edad y asistir a la escuela; b) ser víctima de violencia intrafamiliar, y c) ser soltera con trabajo.

El ministro Pérez Dayán, encargado del proyecto, propuso dar la razón a la CNDH de que la norma impugnada asigna a la mujer el rol de cuidado de las hijas e hijos y que, como consecuencia de tal "estereotipo", provoca que aquellos hombres en la misma situación de vulnerabilidad que las mujeres no gocen del mismo trato preferencial, en violación al principio de igualdad. En este sentido, el ponente consideró que, si bien la medida legislativa persigue la finalidad constitucionalmente válida de acelerar la igualdad sustantiva entre mujeres y hombres, esta no resulta apta ni idónea para alcanzar dicha finalidad, al basarse en estereotipos sobre el género que, por el contrario, terminan por obstaculizar la paridad de derechos entre los

1 “Artículo 27... Asimismo, tendrán prioridad para la admisión en los CACI las hijas e hijos: I. De madres entre 12 y 22 años 11 meses de edad, que comprueben estar inscritas en los niveles básico, medio superior o superior del sistema educativo nacional y que por asistir a la escuela no puedan proporcionar la atención y cuidados necesarios a sus hijas e hijos; II. De madres víctimas de violencia intrafamiliar; III. De madres solteras que requieran la atención de su niña o niño por motivos laborales".

Cuestiones Constitucionales, Núm. 44, Enero-Junio 2021

ISSN: $2448-4881$ 
Esta revista forma parte del acervo de la Biblioteca Jurídica Virtual del Instituto de Investigaciones Jurídicas de la UNAM

sexos. El propósito legislativo, a su juicio, podría atenderse adecuadamente mediante una clasificación neutral, pues lo que hacía verdaderamente necesario el otorgamiento del beneficio radica en la situación de vulnerabilidad o desventaja objetivamente considerada, de ahí que no fuera necesario ni justificable establecer una distinción jurídica entre hombres y mujeres. Adicionalmente, propuso que la norma debía declararse violatoria del principio de interés superior de los menores, pues, de lo contrario, se validaría la posibilidad de no admitir en forma preferencial a un grupo de niñas y niños, bajo el pretexto del género de los progenitores.

Estas consideraciones, que a primera vista parecieran razonables, fueron duramente rechazadas por el resto de las ministras y ministros, entre los cuales hubo quien, incluso, tachó al proyecto de "[defender] la misma igualdad formal que ignora la historia de discriminación y opresión contra las mujeres", de "no [hacer] sino invisibilizar el problema", de "[incurrir] en los estereotipos de género, que dice combatir" e, incluso de "[fortalecer la] cultura machista que debemos desterrar por completo". ${ }^{2}$ El propósito de esta opinión, que intenta ser conciliadora, es mostrar que los anteriores ataques no son más que innecesarios, y que una justa comprensión del reclamo efectivamente planteado, aun cuando tiene que ver con una violación "formal" de igualdad, puede llevarnos a aceptar que una solución como la propuesta en el proyecto - incluir a los hombres - no riñe con una visión estructural de la igualdad. Aun cuando el reclamo de violación al interés superior del menor tiene importancia para la solución del asunto, los fines que inspiraron el diseño de la medida hacen que la respuesta a este dependa, en todo caso, de cómo entendamos el problema de igualdad entre mujeres y hombres para los efectos de la norma. Por eso, solo nos enfocaremos en este último punto.

\section{LA CRÍTICA}

Con la salvedad de algunos matices relevantes de los que luego nos ocuparemos, la postura mayoritaria, que pugna por reconocer la validez de la medida, puede resumirse, primero, en un juicio basado en la estadística que

2 Suprema Corte de Justicia de la Nación. (2021). Contenido de la versión taquigráfica de la sesión pública ordinaria del Pleno de la Suprema Corte de Justicia de la Nación, celebrada a distancia el lunes 8 de marzo de 2021. Disponible en: https://www.scjn.gob. $\mathrm{mx} /$ pleno/secretaria-general-de-acuerdos/versiones-. 
Esta revista forma parte del acervo de la Biblioteca Jurídica Virtual del Instituto de Investigaciones Jurídicas de la UNAM

demuestra, por un lado, la realidad de un grupo significativo de mujeres en condiciones de vulnerabilidad —el de las madres adolescentes que asisten a la escuela, las madres solteras trabajadoras y las madres víctimas de violencia familiar - $\mathrm{y}$, por otro lado, que esta misma condición de vulnerabilidad afecta significativamente más a las mujeres que a los hombres, por lo que, se afirma, la distinción normativa no perpetúa un estereotipo, sino que recoge la realidad. En segundo lugar, y a partir de lo anterior, la postura se constriñe a un juicio de razonabilidad. La medida impugnada - el acceso prioritario - es constitucional porque se encuentra racionalmente conectada con el logro de finalidades legítimas e, incluso imperiosas, al impedir que niñas y mujeres se vean obligadas a abandonar sus estudios para no desatender sus obligaciones de cuidado; procurar que las mujeres obtengan la independencia económica que les facilite salir de situaciones de violencia, y mitigar los obstáculos que dificultan a las madres solteras incorporarse plenamente al mercado laboral.

Frente a la tesis de que la distinción normativa se basa en estereotipos, la mayoría opuso el intento de remediar una realidad estructuralmente desigual. De entrada, esto implica un falso dilema. El estereotipo puede o no conformarse a la realidad, de modo que ambas premisas no necesariamente son excluyentes entre sí. Por eso, la disyuntiva entre realidad o estereotipo no puede ser apropiada para distinguir dos presupuestos de los que puedan derivarse soluciones contrapuestas. Con independencia de esta aclaración, nos parece que responder el problema planteado, preguntándonos primero cuál es la forma correcta de concebir el trato prioritario en favor de las mujeres, es un error. Si la CNDH demanda que los hombres en las mismas condiciones de vulnerabilidad previstas en la norma también participen del beneficio, carece de absoluta relevancia para juzgar esta pretensión sostener, como hace la mayoría, que ese trato preferente no responde a un estereotipo sino a la realidad. Esto se debe a que la posición de las mujeres en la norma no esté, en absoluto, cuestionada.

Como argumento para demostrar lo infundado que es el reclamo de la $\mathrm{CNDH}$, resulta falaz afirmar que la medida impugnada persigue la finalidad legítima de proteger a las niñas y adolescentes que estudian, a las madres solteras que trabajan o aquellas que han sufrido violencia intrafamiliar, por la sencilla razón de que la demandante no opina lo contrario e, incluso, puede estar de acuerdo en eso y, todavía, insistir en su pretensión de igualdad. Es una falacia de la que el propio proyecto no se escapa. Este error, que debería parecer evidente, tiene su origen en una aplicación mecánica del 
Esta revista forma parte del acervo de la Biblioteca Jurídica Virtual del Instituto de Investigaciones Jurídicas de la UNAM

test de igualdad, nunca atenta a la identificación del régimen efectivamente impugnado. En los casos de igualdad, lo que comúnmente se impugna es una exclusión, con el ánimo de ser incluido. El juicio de igualdad y su exigencia de razonabilidad debe aplicarse propiamente a la exclusión. Es decir, habrá que analizar si la medida que conlleva a la exclusión de los sujetos que reclaman estar incluidos está conectada racionalmente con una finalidad legítima. Somos atinados, por suerte, al aplicar el juicio de igualdad en los casos de exclusiones explícitas, es decir, donde se intuye con algo de facilidad o hay evidencia de que el legislador persigue una finalidad más o menos clara al excluir a ciertos sujetos de un régimen donde, por lo general, se recibe un beneficio. Pero en los casos de exclusiones implícitas, comenzamos a dar auténticos palos de ciego que nos llevan a cuestionar falazmente la racionalidad del régimen de beneficio en lugar de la del régimen de no beneficio, que es el efectivamente impugnado. En otras palabras, en casos como este, desorientados, nos preocupamos por demostrar que el trato preferencial a las mujeres es adecuado para protegerlas, en lugar de analizar, como lo pide la demandante, si existe una razón válida para no dar a los hombres el mismo trato.

No es lo mismo buscar incluir a los hombres en la medida a querer excluir a las mujeres. De ahí la importancia de identificar adecuadamente el régimen impugnado de acuerdo con la pretensión efectivamente planteada. Acerca de una medida afirmativa como la que analizamos pueden aducirse dos vicios distintos desde el punto de vista de la igualdad. Un primer vicio es de suprainclusividad. Este conllevaría la negación de que las mujeres o que ciertas mujeres sean merecedoras del beneficio y tildaría a la norma, por tanto, de instituir un mero privilegio. Un segundo vicio es de infrainclusividad que, a diferencia del primero, no cuestiona la posición de quienes participan del beneficio, sino la omisión de no incluir otra categoría que también lo amerita. ${ }^{3}$ Pareciera que el error fundamental de la mayoría, con sus argumentos en favor del derecho de las mujeres al acceso preferencial, consistió en intentar desacreditar un caso de suprainclusividad, cuando en realidad se les planteaba uno diferente, de infrainclusividad normativa.

Sin embargo, sería inapropiado no mencionar que el ministro Laynez Potisek, dentro de la mayoría, dio una respuesta directa al problema efectivamente planteado: "Si hay razón para distinguir entre la protección que se debe dar a una mujer en una situación vulnerable y a un hombre en una situación vulnerable". Su posición fue clara: "aun cuando hay muchos

3 Sobre estos conceptos, Tussman y tenBroek (1949: 348 y 351). 
Esta revista forma parte del acervo de la Biblioteca Jurídica Virtual del Instituto de Investigaciones Jurídicas de la UNAM

hombres en las situaciones que se puedan establecer en otras infracciones impugnadas, la realidad es que las cifras apuntan a que en las tres problemáticas hay un impacto desproporcional en la mujer, lo que justifica esta priorización que hace el legislador". Nos parece que, a falta de traducir esta desproporcionalidad en una condición más cualitativa que cuantitativa, el inconveniente de esta postura es que cae en la falacia de convertir cantidad en calidad. Si se tienen cuatro infantes que alimentar, y solo uno de ellos es varón, sería poco razonable administrar la comida bajo un criterio de justicia que favorezca a las niñas por la sola circunstancia de que ellas sean más, porque no hay razones para sostener que un niño sienta menos hambre que una niña, con independencia de cuántas niñas y cuántos niños existan en el mundo o en el específico escenario del problema de distribución.

No queremos decir que las niñas no puedan tener nunca preferencia sobre los niños en la distribución justa de algún recurso escaso, sino solo que el hecho de que ellas sean más no justifica que deban tenerla en un escenario en el que lo que importa es la necesidad de comer. Por eso tampoco estamos diciendo que atender a través de medidas no neutrales aquellos problemas en los que las mujeres son desproporcionalmente las más agraviadas sea irracional, sino que lo irracional es asumir que, cuando esa desproporción no se refiera más que a diferencias de cifras, entonces hay un deber de discriminar como si tales cifras se trataran de cualidades de las personas. Sería también impropio pasar inadvertida la fórmula del ministro Gutiérrez Ortiz Mena de que los hombres "están mejor posicionados frente a esos escenarios límite". Pero este aserto, si se le concede al ministro no caer en una falacia de generalización indebida, se traduciría en un argumento de la misma naturaleza cuantitativa que el del ministro Laynez, pero destinado a reducir, sin desvanecerlo, el universo de los hombres que podrían merecer el trato preferente.

Parecería que estamos, como dice la mayoría, pasando por alto una "visión estructural de la igualdad", aquella que, en palabras de Roberto Saba, "en lugar de tomar como elemento único de juicio la relación de funcionalidad entre la categoría escogida para hacer diferencias y la actividad regulada, considera relevante la situación de la persona individualmente considerada pero como integrante de un grupo sistemáticamente excluido" (Saba, 2005: 126). Queremos pensar que no es el caso. El acceso prioritario intenta paliar causas específicas que pueden explicar el fenómeno de sometimiento y la exclusión sistemática que padece el grupo de las mujeres 
Esta revista forma parte del acervo de la Biblioteca Jurídica Virtual del Instituto de Investigaciones Jurídicas de la UNAM

en nuestro país. La visión estructural de la igualdad, podríamos decir, ha quedado ya recogida en la implementación de esta medida, la cual no intenta ni podrá jamás ser anulada por quien diga que se queda corta en cuanto al grupo de personas al que se dirige.

La característica tensión que se da entre la igualdad formal o como no discriminación y la igualdad estructural al administrar posiciones valiosas, echando mano, en parte, de una acción afirmativa, se traduce, concretamente, en la disputa entre el criterio estrictamente funcional de administración de las posiciones y un criterio no funcional (el sexo, el origen étnico, una condición de discapacidad) pero que favorece la inclusión de un grupo socialmente desaventajado. Es un conflicto dilemático porque, en última instancia, supone que un integrante del grupo "no sometido", que satisfizo el criterio funcional de la medida, sea desplazado por quien no satisfizo totalmente este criterio, pero que pertenece al grupo "sometido". Por ejemplo: el estudiante que alcanzó el puntaje mínimo general de ingreso a la universidad pierde su lugar en favor de la estudiante que obtuvo un puntaje menor y solo porque esta última es mujer. En resumen, la igualdad estructural exigiría que, en posiciones desiguales en cuanto exclusión o marginalidad, se favorezca a los integrantes del grupo socialmente desaventajado por encima de (algunos de) los que pertenecen al que socialmente no es así, aunque esto no sea necesariamente racional desde el punto de vista del propósito del ámbito administrado.

Con motivo de la acción afirmativa que específicamente analizamos, parecería surgir una tesis que, por decirlo casi coloquialmente, nos saca de la teoría: en nombre de la igualdad estructural, también debiéramos tratar diferente a quienes se encuentran en un mismo grupo desaventajado. Una tesis que sería útil para justificar acciones afirmativas a favor de grupos que atraviesan por dos o más categorías históricamente marginadas (ser mujer, indígena y pobre, por ejemplo), en el sentido de tener que rechazar, por ser contrarios a la idea de igualdad estructural, por ejemplo, los reclamos provenientes de varones indígenas para ser considerados dentro de una política que solo favorezca a las mujeres indígenas el acceso al mercado laboral. No es una tesis cómoda de admitir, porque, como ya dijimos, no se explica por esta concepción académica y un tanto maniquea de favorecer la igualdad entre un grupo subordinado frente al resto de la sociedad que históricamente así lo ha mantenido. Pero también porque si, en el ejemplo, los varones indígenas no son objeto de ninguna otra medida encaminada a optimizar su situación, aunque sea neutral, esta acción afirmativa se traduciría en una 
Esta revista forma parte del acervo de la Biblioteca Jurídica Virtual del Instituto de Investigaciones Jurídicas de la UNAM

especie de trato que equipararía a los indígenas varones y al resto de la sociedad, y que difícilmente podríamos justificar desde la misma visión estructural de la igualdad. En un escenario como el descrito, los indígenas varones tendrían motivos, nos parece, muy justos para incomodarse, sin que esto implique necesariamente que estén en desacuerdo con que el Estado continúe apoyando a las mujeres de su grupo.

Desde luego, "ser varón" no puede compararse con "ser indígena", en términos de igualdad estructural. Solo en una realidad alternativa pudiera estimarse que "ser varón" constituye una categoría en condición de exclusión o inferioridad. Pero esto no quiere decir que el planteamiento de justicia de los varones en el caso analizado sea esencialmente distinto al de los varones indígenas en el ejemplo anterior. Al igual que ellos, no reclaman su inclusión por el hecho de "ser varones" nada más, sino por la situación vulnerable de tener a su cargo el cuidado de un hijo o hija, en edad para estudiar, cuando sufren violencia intrafamiliar o cuando se disponen a trabajar, siendo solteros. Es entendible, pues, que les incomode ser colocados en la misma fila que a las mujeres y los hombres que no atraviesan por estas situaciones. Si bien estas últimas circunstancias pudieran difícilmente identificar a un grupo como sometido o bajo exclusión histórica, justificante del trato privilegiado que supone una acción afirmativa, tampoco se trata de privilegiar simplemente a una persona por "ser varón", caso que sí constituiría un despropósito claro para la igualdad estructural.

No debe perderse de vista también que, en el caso del acceso prioritario, la categoría "ser mujer" no es la relevante para obtenerlo, sino ser madre en edad para estudiar, ser madre que sufre violencia doméstica y ser madre soltera que busca trabajar. Por supuesto que ser mujer es, en términos de la norma, una condición sine qua non para actualizar el supuesto de hecho, pero, cuando afirmamos que no es relevante para efectos de la acción afirmativa, queremos decir que la condición de ser mujer no identifica a una persona en automático como perteneciente al grupo vulnerable al que la medida busca favorecer. Si observamos con cuidado - la mayoría, curiosamente, hizo notar este aspecto-, podemos apuntar que la medida distingue incluso entre un grupo de "mujeres (madres) no vulnerables" (y también de "hombres [padres] no vulnerables"), y un grupo exclusivo de "mujeres (madres) vulnerables", para los propósitos de la norma. En otros términos, pues, "ser mujer" no es una condición necesaria y suficiente para pertenecer al grupo en desventaja a igualar. Por eso, desde esta perspectiva meramente lógica, que no pierde de vista el horizonte de la igualdad estructural, no 
Esta revista forma parte del acervo de la Biblioteca Jurídica Virtual del Instituto de Investigaciones Jurídicas de la UNAM

resulta tan obvia la exclusión de los "padres" del acceso preferente bajo análisis, pues "ser hombre" no será la categoría que identifica al grupo en ventaja estructural frente al cual se busque la igualdad, mientras que "ser mujer" no sea suficiente para pertenecer a la categoría desaventajada.

El análisis puramente lógico nos lleva a definir como cuestión clave a examinar: si "ser mujer" debe ser una condición necesaria para pertenecer a la categoría en desventaja, caso en el que excluiríamos a los hombres que tuvieran las mismas condiciones de vulnerabilidad identificadas, o bien si poseer estas condiciones de vulnerabilidad debe ser necesario y suficiente para pertenecer a la categoría en desventaja que favorece la norma, caso en el que incluiríamos a las personas que cumplan este requisito, con independencia de su sexo. El hecho de que la condición "ser mujer" no sea en todo caso suficiente para identificar quién pertenece, según la norma, a la categoría en desventaja a igualar, sino que se le deben sumar otras condiciones a las que también pueden enfrentarse el grupo de los hombres, podría restar convicción al enunciado de que la medida sigue estrictamente un prolegómeno de igualdad estructural en el sentido de que se funde en la identificación de "las mujeres" como grupo al que se le deban reconocer prerrogativas que sería impensable atribuir al grupo de "los hombres". Luego, si afirmamos terminantemente que en este caso no sería lícito favorecer a un "padre", pudiéramos no estar haciendo otra cosa más que discriminar terminantemente, en el sentido prohibido por el artículo 1o. de la Constitución, por razón de sexo.

Conviene ser reiterativo: los argumentos anteriores se encuentran dirigidos más bien al criterio de administración de la medida, que a la medida en sí misma, por lo que no buscan anular esta acción afirmativa ni, digamos, las acciones afirmativas en general. El punto es que, como cualquier otra medida que intenta solucionar una injusticia social, las acciones afirmativas no están exentas de ser infrainclusivas o suprainclusivas. Podría impugnarse que la medida impugnada en esta acción tiene un vicio de suprainclusividad (admite personas no vulnerables) porque reconoce igualmente prioridad para la admisión de sus hijos e hijas en los CACI a las madres solteras adineradas o de familias pudientes, que podrían costear cómodamente un servicio privado de guardería o de niñera, o bien un vicio de infrainclusividad (deja fuera personas igualmente vulnerables), porque excluye a las mujeres trabajadoras no solteras pero cuyo esposo ha adquirido una discapacidad que le impide incluso cuidar a sus hijos o hijas, o a las mujeres indígenas, o a las mujeres con alguna discapacidad, o también, simplemen- 
Esta revista forma parte del acervo de la Biblioteca Jurídica Virtual del Instituto de Investigaciones Jurídicas de la UNAM

te, porque, como lo adujo la $\mathrm{CNDH}$, no tomó en cuenta a los hombres que pueden atravesar por las mismas situaciones de vulnerabilidad.

Por más natural que parezca este ejercicio analítico, y del cual no queda exenta cualquier medida que, con base en un trato desigual, tiende a conseguir una sociedad más justa, no debe sorprendernos, allende se le confunda, que se le descalifique de raíz. La escasa práctica analítica, asociada a un prejuicio o temor hacia lo "formal", puede tender, como en el caso, a dotar a las medidas afirmativas a favor de las mujeres de un blindaje irracional que refleja cualquier crítica al modo en que son administradas como un ataque hacia la mujer o una forma de invisibilizar su histórica y nefasta marginación. Se corre el riesgo de terminar convirtiendo estas medidas en verdaderos tabús o constructos en los que la razón queda vedada. Todo esto a pesar de que, en principio, la preferencia al grupo de mujeres específico no esté siquiera en discusión. Las medidas afirmativas son óptimas cuando, efectivamente, dividen a la sociedad entre quienes sí se encuentran y quienes no se encuentran en condiciones de desigualdad estructural, y requieren ser ajustadas cuando, por prejuicios o simple descuido, traten diferente a las personas que se hallan en las mismas condiciones de desigualdad estructural o igual a quienes no se hallen en estas mismas condiciones. Si a los juicios que justifican la necesidad de estos ajustes se les llama "formalistas", es innegable la contribución de los juicios formales o analíticos al propósito mismo de la igualdad estructural.

La violación a la igualdad aducida por la $\mathrm{CNDH}$ es fundada, mientras nadie pueda negar la existencia de hombres que compartan las mismas condiciones de vulneración que recogen las fracciones impugnadas y "ser mujer" sea insuficiente para identificar la categoría a igualar estructuralmente. Un problema aparte son las consecuencias que tendría una declaración de esta naturaleza en un medio de control abstracto (como lo es la acción de inconstitucionalidad), las cuales están lejos de ser evidentes. Los malentendidos que tenemos acerca del concepto formal de igualdad nos han privado, hasta el momento, de un debate sugestivo en el Alto Tribunal. Nuestra opinión al respecto es que, desde luego, no toda violación formal a la igualdad puede derivar en una invalidez general de la norma, pues subyacería a nuestro juicio la condición irracional de que la actividad legislativa debe ser perfecta: si no es perfecta no es válida. Con excepción tal vez de las que contienen derechos humanos, todas las normas distinguen, y como no lo hacen a la perfección — como se aspira que sean los tipos penales-, expulsar normas del orden jurídico por vicios formales de igualdad — de 
Esta revista forma parte del acervo de la Biblioteca Jurídica Virtual del Instituto de Investigaciones Jurídicas de la UNAM

infrainclusividad o de suprainclusividad - haría imposible no sólo la función legislativa sino también la jurisdiccional. Deberían invalidarse solo aquellas normas que distingan por motivos discriminatorios o cuya incidencia no podría escaparse de las miras de un legislador razonablemente cuidadoso y cuya importancia de ser eficientemente regulados, por seguridad jurídica, torne insuficiente la justicia de los casos concretos (es decir, la integración del derecho a partir de juicios por analogía o mayoría de razón a cargo de las y los jueces).

A pesar de esta regla, el proceder en el caso analizado es poco claro. Hasta aquí, es decir, llegada la conclusión de que existe una violación formal a la igualdad, es donde sí pareciera relevante analizar si la distinción entre "madres" y "padres" es o no un estereotipo. Todavía más difíciles, sin embargo, creemos, son de explorar las consecuencias que tiene el reconocimiento de validez de la norma cuando el vicio de igualdad aducido no se estudió en sus méritos, es decir, como en este caso, en el que el trato prioritario se encontró justificado, sin aún averiguarse — con la salvedad de los ministros Laynez y Gutiérrez - si existía una razón válida para que los hombres igualmente vulnerables no lo tuvieran. El ejercicio es más complejo, pues afirmar, por ejemplo, que es posible otorgar, en los casos concretos, un amparo a cualquiera que exija un trato prioritario, conforme a la razón de ser de la norma impugnada, sea un hombre o una mujer no incluida en la medida, pasa por contestar qué tanto quienes juzgan pueden resistir a ser tachados, hoy, de "formalistas", por preferir la decisión técnicamente correcta, a la más popular.

\section{BIBLIOGRAFÍA}

SABA, Roberto. (2005). (Des)igualdad estructural. Revista Derecho y Humanidades, Facultad de Derecho Universidad de Chile. Núm. 11.

Suprema Corte de Justicia de la Nación. (2021). Contenido de la versión taquigráfica de la sesión pública ordinaria del Pleno de la Suprema Corte de Justicia de la Nación. Disponible en: https://www.scjn.gob. $m x /$ pleno/secretaria-general-de-acuerdos/versiones-taquigraficas.

Tussman, Joseph y TenBroek, Jacobus. (1949). The Equal Protection of the Laws, California Law Review. Vol. 37. Núm. 3. 